\title{
UN ANÁLISIS DE LOS PROFESIONALES EN ECONOMÍA DE LA UNIVERSIDAD MILITAR NUEVA GRANADA: MERCADO LABORAL Y COMPETENCIAS*
}

\author{
JOSÉ ISRAEL BAUTISTA RAMÍREZ", JUAN CAMILO RINCÓN TORRES \\ \& DAVID ANDRÉS CAMARGO MAYORGA \\ UNIVERSIDAD MILITAR NUEVA GRANADA
}

Recibido/ Received/ Recebido: 26/04/2012 - Aceptado/ Accepted / Aprovado: 02/05/2013

\begin{abstract}
Resumen
El presente artículo presenta un diagnóstico de la formación y desarrollo profesional de los economistas egresados de la Universidad Militar Nueva Granada, a partir de 58 encuestas a exalumnos. Se contrasta dicha información con los requerimientos específicos que hace el mercado laboral a través del Consejo de Empresas Americanas (CEA) y el Grupo Social y Empresarial de la Defensa (GSED). El estudio arroja coincidencias significativas en torno a las necesidades y requerimientos que se demandan, teniendo en cuenta aspectos fundamentales como la inserción de asignaturas relevantes, la inclusión laboral, el manejo de conocimientos específicos y en términos generales la consolidación de economistas fuertemente instruidos en competencias argumentativas, propositivas, interpretativas y analíticas.
\end{abstract}

Palabras clave: Mercado laboral, Competencias profesionales, Egresados, Educación superior.

\section{AN ANALYSIS OF ECONOMISTS FROM THE NUEVA GRANADA MILITARY UNIVERSITY: LABOR MARKET AND SKILLS}

\begin{abstract}
This article presents an assessment of training and professional development of economists from Nueva Granada Military University, based from 58 surveys to graduates. This information is compared with the specific requirements that labor market asks through the American Business Council (CEA for its acronym in Spanish) and the Defense Social and Business Group (GSED for its acronym in Spanish). The study shows significant matches around needs and requirements demanded, considering basic aspects such as inclusion of important subjects, labor inclusion, handling specific knowledge and broadly consolidation of economists strongly educated for argumentative, purposeful, interpretive and analytic skills.
\end{abstract}

Keywords: Labor market, Professional skills, Graduated, Higher education.

Artículo de resultados de investigación derivado del proyecto ECO 715: "El perfil de los egresados de Economía de la Universidad Militar Nueva Granada. Un análisis a partir del mercado laboral", financiado por la Universidad Militar Nueva Granada en el año 2011.

* Economista y Especialista en Docencia Universitaria de la Universidad Militar Nueva Granada, Magister en Planeación Socioeconómica de la Universidad Santo Tomás de Aquino. Docente investigador del programa de Economía y miembro del Grupo CIE de la Universidad Militar Nueva Granada. Correo electrónico: jose.bautista@unimilitar.edu.co

*** Economista Universidad Militar Nueva Granada. Joven investigador Grupo CIE de la Universidad Militar Nueva Granada. Correo electrónico: juanc.rincon@gmail.com

*nst Economista y estudiante Maestría en Economía de la Pontificia Universidad Javeriana. Docente investigador del programa de Contaduría Pública de la Universidad Militar Nueva Granada. Correo electrónico: david.camargo@unimilitar.edu.co 


\title{
UMA ANÁLISE DOS PROFISSIONAIS EM ECONOMIA DA UNIVERSIDADE MILITAR NOVA GRANADA: MERCADO TRABALHISTA E COMPETÊNCIAS
}

\begin{abstract}
Resumo
O presente artigo apresenta um diagnóstico da formação e desenvolvimento profissional dos economistas formados na Universidade Militar Nova Granada, a partir de 58 questionários aplicados a ex-alunos. Contrasta-se dita informação com os requerimentos específicos que faz o mercado trabalhista por parte do Conselho de Empresas Americanas (CEA) e o Grupo Social e Empresarial da Defesa (GSED). O estudo revela coincidências significativas em torno das necessidades e requerimentos exigidos, considerando aspectos fundamentais como a inserção de matérias relevantes, a inclusão laboral, o manejo de conhecimentos específicos e, em termos gerais, a consolidação de economistas fortemente instruídos em competências argumentativas, propositivas, interpretativas $e$ analíticas.
\end{abstract}

Palavras chave: Mercado laboral, Competências profissionais, Ex-alunos, Educação superior.

Bautista, J., Rincón, J. \& Camargo, D. (2013) Un análisis de los profesionales en economía de la Universidad Militar Nueva Granada: mercado laboral y competencias. En: Revista de la Facultad de Ciencias Económicas de la Universidad Militar Nueva Granada. rev.fac.cienc. econ, XXI (1).

JEL: A11, A2, A22.

\section{Introducción}

"El cuerpo de conocimiento económico es un elemento esencial en la estructura de la civilización humana, es la base sobre la que se construyeron el industrialismo moderno y todos los avances morales, intelectuales, tecnológicos y terapéuticos de los últimos siglos. Está con los hombres, ya sea que hagan uso adecuado del rico tesoro que este conocimiento les provee o que lo dejen sin utilizar. Pero si ello no lo aprovechan y desoyen sus enseñanza y advertencias, no anularán la economía; desecharán a la sociedad y a la raza humana" (Von Mises, 2009).

La enseñanza de la economía en la educación superior reviste importancia, porque ofrece a los estudiantes herramientas intelectuales para la comprensión de fenómenos sociales y la solución de problemáticas derivadas de estos. Calderón et al. (2008) plantea que siendo la economía una forma concreta de pensar el mundo y sus interrelaciones, expresa respeto por la evidencia empírica. De manera que la instrumentalización matemática de la economía no la hace necesariamente fría y alejada de lo social, por el contrario son los instrumentos los que están al servicio del diagnóstico económico y no al contrario.

Ahora bien, la formación académica de los economistas suele propender por la instrucción en áreas de conocimiento fundamentadas a partir de los requerimientos profesionales nacionales e internacionales y el desarrollo de competencias analíticas, interpretativas, argumentativas y propositivas como reflejo del compendio teórico y práctico del proceso académico en el estudio de su profesión. Por su parte, el mercado laboral plantea necesidades genéricas y específicas que deben ser objeto de interés constante por parte de quienes buscan una ubicación profesional importante. Así, el presente documento presenta un diagnóstico de la formación y desarrollo profesional de los economistas neogranadinos ${ }^{1}$, esbozando aquellas características de formación

1 Economistas graduados de la Universidad Militar Nueva Granada - UMNG. 
contrastadas con los requerimientos del Consejo de Empresas Americanas (CEA) y el Grupo Social y Empresarial de la Defensa (GSED). La construcción del estudio empírico contemplo la elaboración de 58 encuestas a exalumnos graduados del programa de economía. De ahí que este ejercicio examine puntos clave en la identificación de fortalezas y debilidades del programa de economía de la Universidad Militar Nueva Granada (UMNG), que lleven a la consolidación de egresados competitivos, fuertemente instruidos y competentes para el trabajo.

Este documento consta de siete secciones siendo ésta la primera. En la segunda parte se trata la relación entre educación e inserción laboral de los recién egresados, en la tercera se habla sobre competencias, en la cuarta se explica la metodología empleada, en la quinta se presentan los resultados, en la sexta se hace una discusión de los hallazgos, y en la sección final se hacen algunas consideraciones finales.

\section{Educación e inserción laboral de los recién graduados}

De las transformaciones del sistema educativo la que tiene que ver con la calidad ha sido de las más importantes en América Latina, lo que condujo desde los años noventa a reorientar esfuerzos en su búsqueda (Carlson, 2000). En tal sentido, son la acreditación y la evaluación externa formas de conseguirla, aunque no las únicas, lo cual implica que las instituciones educativas deben ser evaluadas por externos, siendo esta práctica de acreditación conveniente y necesaria para plantear planes de mejora institucional. Según Aguila (2005) la calidad ha provocado que la universidad (llas licenciaturas y programas académicos de pregrado) se vea obligada a participar en la carrera por la subsistencia, en donde un factor de extrema importancia para mantenerse y ganar es ser competitiva, y esto básicamente consiste, en primer lugar, en poseer calidad al cumplir los criterios solicitados por quien evalúa, y en segundo, que la institución educativa sea reconocida a partir de su acreditación, lo cual ofrece un "good will".

Un aspecto relevante en el entorno educativo es la satisfacción de los agentes, lámense empleadores, docentes, egresados o comunidad en general, que son quienes componen el sistema en el cual se desenvuelven los profesionales de una rama del saber. Según Cardoso \& Giraldo (2003) la educación superior debe estar enmarcada en tres aspectos principales: (i) los fines educativos, (ii) los objetivos de la institución, y (iii) la satisfacción de las necesidades de los estudiantes que son en ultimas los clientes. Sobre este último punto existen discrepancias en la literatura, por ejemplo, Vasconez \& Trujillo (2004) explica que los clientes son las organizaciones que demandan personal calificado. De su demanda depende el mercado laboral. Por lo tanto son ellos los que definen los requerimientos de formación en términos de competencias que se adecuen al desempeño que esperan de los egresados universitarios en sus organizaciones.

En el argumento de Vasconez \& Trujillo (2004), el enfoque que se debe tomar para conocer cuáles son las principales fortalezas y debilidades del egresado, es precisamente el de identificar los requerimientos de las empresas. A este respecto, Lora \& Ñopo (2009) muestran en su estudio que hay una debilidad evidente en Argentina, Bolivia, Chile, Colombia y México por la no concordancia entre las expectativas que los estudiantes de economía tienen sobre el mercado laboral y las demandas reales de economistas por parte de los sectores económicos de tales países.

Bajo estas circunstancias, Zúñiga (2003) plantea que a la academia se le exige identificar los requerimientos para el desempeño laboral, como insumo fundamental para la preparación de acciones de formación y desarrollo que fortalezcan las capacidades de las personas que educa, ampliando sus posibilidades de inserción laboral y social exitosa, porque de acuerdo con Tedesco et al. (2008) los egresados sienten que las habilidades y competencias que adquirieron en las universidades, no son las adecuadas para el mercado. Frente a tal problemática, el SENA $(2003,7)$ concluye que es necesario "un modelo basado en normas de competencia laboral, el cual contribuya a la cualificación del mercado de trabajo, fortaleciendo la satisfacción de vinculación de las personas al mundo laboral y orientando la formación profesional hacia accio- 
nes pertinentes y flexibles, como fundamento de calidad y equidad."

Por todo esto, es indispensable reconocer que un modelo de formación por competencias es relevante en la medida que equipa a los egresados para una inserción laboral menos traumática, aumentando la satisfacción del entorno y de los mismos individuos que se han educado, acortando las diferencias entre lo que esperan del mercado laboral y lo que en la realidad económica se les demanda. En tal sentido, García-Montalvo (2005), en su estudio sobre la inserción laboral de los graduados universitarios en España, señala que hay tres factores fundamentales que explican el rechazo de un trabajo por parte de un joven profesional universitario: (i) que exista poca relación del trabajo con sus estudios, (ii) que el salario no sea adecuado y (iii) que el trabajo requiera un nivel profesional inferior al de estatus de graduado universitario.

En la misma línea Teichler (2005) citado por ANECA (2009) llega a concluir que existen cinco criterios principales para determinar el éxito en el empleo de quienes recién egresan de la educación superior y se enfrentan las condiciones del mercado: (i) una transición suave al empleo conseguido, lo que implica inmediatez entre el grado y el acceso al trabajo, y corto tiempo y mínimo esfuerzo de búsqueda; (ii) una proporción baja de desempleo; (iii) una proporción baja de empleo precario (empleo ocasional, de corta duración, a tiempo parcial); (iv) mejor remuneración en comparación con los no graduados o alta proporción de graduados con un empleo adecuado; (v) estrecha relación entre el campo de estudios y el empleo conseguido o la pertinencia para el trabajo de los conocimientos aprendidos durante el proceso de formación.

En ese orden de ideas, son la pertinencia y la calidad, junto a la internacionalización, en palabras de la UNESCO, tres aspectos clave que determinan la posición estratégica de la educación universitaria. Siendo el grado de pertinencia social de un programa o institución, una medida del impacto social que genera, por el flujo de repercusiones y de transformaciones de sentido que se producen objetivamente en la sociedad y en el entorno (Aguila, 2005).

\section{Competencias}

En la economía de mercado, lo que se espera es que los recién graduados hayan desarrollado competencias que los habilitan para el trabajo. En tal sentido Periáñez et al. (2010) afirman que las competencias de los economistas pueden ser valoradas en la medida que satisfagan a los empleadores. Por eso los estudios realizados por los empleadores han estado enfocados en los métodos y criterios de reclutamiento y en las competencias de los graduados y los posibles requerimientos futuros de mano de obra con habilidades específicas (Schomburg, 2004).

En Colombia, el SENA (2003) realizó una investigación general sobre la metodología de elaboración de normas de competencia laboral, y aunque ésta institución es la encargada de la formación técnico-profesional en el país, encontró ciertas similitudes en la demanda laboral de profesionales y tecnólogos.

De donde se desprende que "los modelos de competencia son un insumo fundamental para las entidades de formación ya que actualizan la respuesta de formación, mejoran los procedimientos de orientación ocupacional y profesional, mejoran la calidad, pertinencia y flexibilidad de la oferta educativa" (SENA, 2003, 8).

Asimismo está la formación en el puesto de trabajo, que según Márquez (2005) comprende la enseñanza directa ahí mismo, concatenando la formación que se adquirió como aprendiz en el salón de clases, con el tiempo de experiencia práctica en el lugar de trabajo (práctica profesional).

En consecuencia, uno de los elementos que ha transformado la educación profesional es la incorporación de las competencias para los programas académicos de pregrado, eso sí con una inclinación hacia las necesidades de la demanda laboral. Esto implica que en la sociedad actual, como sociedad del conocimiento, se les exija a los trabajadores que tengan una formación continua, donde el aprender a aprender como competencia, le da la habilidad al individuo para iniciar y persistir en el aprendizaje, 
gestionando el tiempo y la información de manera eficaz, ya sea en grupos de trabajo o de forma individual. Además de potenciar el autoaprendizaje, entendido como la habilidad para buscar, asimilar y compartir nuevos conocimientos que potencien el desarrollo de la persona y del profesional. Así, el desarrollo de habilidades adicionales y la aprehensión de nuevo conocimiento para el trabajo, tiende a recaer sobre el individuo.

Sin lugar a dudas es difícil definir la palabra competencia, cuando este concepto en sí mismo incluye otros conceptos como lo son capacidad, cualificación, aptitud, destreza. Sin embargo como lo describen Periáñez et al. (2010) se pueden distinguir dos tipos de competencias: (i) competencias específicas, que son destrezas y conocimientos relativos a cada área de conocimiento o profesión, y (ii) competencias genéricas, las cuales son destrezas y conocimientos compartidos por la mayoría de ámbitos de conocimiento y profesiones.

Por tal razón es difícil abordar el tema del trabajo y el mercado laboral sin tener algunas nociones sobre la definición de competencias. Según Gallard (1995) la noción de competencia, se encuentra en medio del saber y de las habilidades concretas, por lo cual la competencia es intrínseca de la acción, y exige a la vez conocimiento. Flores (2007) define la competencia como una mezcla de atributos, en relación a conocimientos, habilidades, actitudes y responsabilidades, convirtiéndose en los resultados de un programa formativo, es decir la formación del egresado. Se requiere que la educación superior según Vasconez \& Trujillo (2004), sirva entonces para formar individuos en un conjunto amplio de competencias que incluyan los conocimientos, pero también las actividades y las actitudes del mundo laboral.

\subsection{Competencias vocacionales}

Las competencias vocacionales se refieren directamente al ejercicio de la profesión, es decir al campo de aplicación de los conocimientos adquiridos, en ese aspecto entrarían tales competencias a evaluar la eficiencia de realizar actividades por parte del economista, como la investigación económica, el análisis de los resultados obtenidos, la proyección de resultados, la creación de políticas públicas, entre otras. Según Pérez (2010) se trata de las habilidades del individuo, tales como: (i) capacidad de adaptación, (ii) trabajo en equipo, (iii) saber estar, y (iv) motivación.

\subsection{Competencias transversales}

Son las competencias transversales o genéricas las que pueden ser compartidas entre diferentes carreras. Se relacionan tanto con las actitudes y los valores del saber ser y saber estar, lo que en economía se conoce como normativa. Se relacionan además con los procedimientos del saber hacer como competencias transferibles fuera de la carrera.

Beneitone et al. (2007) define siguiendo el manual de Tuning, que las competencias transversales son destrezas:

- En la comunicación oral y escrita en, por lo menos, dos de los idiomas oficiales de la Unión Europea.

- En la resolución de problemas, en relación con información cualitativa y cuantitativa.

- Numéricas y de cálculo, incluyendo aspectos tales como análisis de error, estimaciones de orden de magnitud y uso correcto de unidades.

- En la búsqueda de información, en relación con fuentes de información primarias y secundarias, incluyendo el uso de ordenadores para búsquedas en línea.

- En las nuevas tecnologías de la información, tales como procesamiento de datos y hojas de cálculo, registro y almacenamiento de datos.

- En comunicación a través de internet, etc.

- Interpersonales, asociadas a la capacidad de relación con otras personas y de trabajo en grupo

- De estudio necesarias para continuar el desarrollo profesional.

La UMNG basada en el Proyecto Tuning (Beneitone et al., 2007) ha venido promoviendo la formación profesional de economistas, con base en $10 \mathrm{com}-$ petencias genéricas: (i) capacidad de abstracción, análisis y síntesis; (ii) capacidad de comunicación en un segundo idioma; (iii) habilidades en el uso de las tecnologías de la información y de la comunicación; 
(iv) capacidad de investigación; (v) capacidad creativa; (vi) capacidad para identificar, plantear y resolver problemas; (vii) capacidad de trabajo en equipo; (viii) capacidad de motivar y conducir hacia metas comunes; (ix) capacidad para formular y gestionar proyectos; $\mathrm{y}(\mathrm{x})$ compromiso ético.

\subsection{Competencias específicas}

Las competencias relacionadas con los conocimientos reciben el nombre de específicas, es decir, el saber y las habilidades propias de la carrera. Estas no son transferibles fuera del ámbito profesional vinculado al área de conocimiento. En este aspecto, el programa de economía de la UMNG, ha especificado las principales competencias que el economista neogranadino debe desarrollar a lo largo de su proceso de formación en las siguientes áreas de conocimiento:

- Competencias de análisis microeconómico: de diagnóstico y evaluación de problemas y oportunidades de los agentes, aplicación y contextualización técnica de modelos para explicar la realidad $e$ investigación efectiva de problemas de optimización y asignación de recursos.

- Competencias de análisis macroeconómico: de diagnóstico, evaluación e investigación de problemas macroeconómicos, aplicación y contextualización técnica de modelos.

- Competencias en diseño de modelos: de diagnóstico de problemáticas para el diseño de modelos explicativos de la realidad. Evaluando apropiadamente a partir de los modelos la interrelación de las variables económicas e investigando de manera rigurosa teorías estadísticas para el diseño de estos.

- Competencias en proyectos: de diagnóstico de problemas económicos para la formulación de proyectos. Evaluación financiera, económica, social y ambiental de estos, de manera técnica, además de proyectos de inversión, y aplicación adecuada de estos a la realidad económica a través de su ejecución.

- Competencias en defensa y seguridad nacional: de evaluación eficiente de posibles alternativas a problemáticas del sector defensa y de seguridad nacional. Pronostico racional del comportamiento y desarrollo de variables relevantes en el sector defensa y de seguridad nacional e investigación sobre economía de la defensa nacional.

\section{Metodología}

Para lograr los objetivos propuestos se realizó un estudio exploratorio. Por un lado, se hizo un muestreo no probabilístico donde se efectuaron 58 encuestas dirigidas a profesionales egresados de economía de la UMNG de distintas promociones y rangos de edad. El instrumento de recolección de información constó de 24 preguntas que a groso modo permitieron esbozar las características, necesidades y falencias del programa reflejadas en la formación ofrecida y el escenario laboral. Las encuestas se sistematizaron en Excel realizando verificación y validación de la información en contraste con la base de datos de egresados suministrada por la División de Registro Académico de la UMNG. Se utilizaron estadísticas descriptivas para presentar los resultados de tales encuestas.

Por otro lado, se contó con la colaboración del GSED y el CEA. El primero de estos grupos, conformado por 18 entidades adscritas y vinculadas al Ministerio de Defensa Nacional dentro de las que está la UMNG. Como centro corporativo encargado de dirigir y orientar las políticas de las empresas del grupo, el GSED tiene como objetivo el reconocimiento por parte de la Fuerza Pública como un sistema estratégico de apoyo logístico, generador de bienestar (educación, vivienda, recreación y salud) y de apoyo a la seguridad ${ }^{2}$. La segunda colaboración la ofreció el CEA, el cual contribuyo de primera mano a la determinación de los requerimientos específicos por parte del mercado laboral. La pertinencia de estos resultados radica en la importancia que tiene el CEA, quien promueve los intereses de las compañías de capital norteamericano en Colombia en sectores de tecnología, seguridad industrial,

2 Información extraída de: http://www.gsed.gov.co/irj/portal/GSED 
consultorías y eléctricos, lo que ha permitido saber de primera mano cuáles son las características relevantes respecto de un potencial mercado de trabajo para los economistas de la UMNG.

Con el fin de conocer las necesidades del mercado laboral se construyó una encuesta dirigida al GSED $y$ al CEA, abordando 4 preguntas principales que permitieron el acceso a información primaria sobre características específicas de los requerimientos que estas instituciones tienen para la contratación de profesionales en economía, teniendo en cuenta el cargo, el área funcional, la competencia equivalente y las competencias del egresado.

\section{Resultados}

La muestra poblacional se tomó a partir de rangos de edad quinquenales. Se encontró que el 30\% de la población encuestada estaba ubicada entre 36 y 45 años y que el $20 \%$ estaba entre los 26 y 30 años de edad (ver Ilustración 1), lo cual resultó un indicativo de que la información recogida provenía de profesionales ubicados de forma estable en el mercado laboral.

Ilustración 1. Rangos de edad de economistas neogranadinos $^{3}$



La proporción de hombres y mujeres egresados encuestados fue equivalente, es decir, fue de 50:50. Su estado civil mostró que el 51,72\% estaban casados, en tanto que el 43,1\% tenían la condición de solteros.

Aproximadamente el 25\% de la muestra obtuvo su título antes del año 2000 y solo el $40 \%$ manifestó poseer matricula profesional. Adicionalmente, el 80,39\% estaba empleado, mientras el 19,61\% se encontraba cesante. Lo cual concuerda con las cifras presentadas por Duque \& Ortiz (2011) para graduados de economía de la UMNG entre 1982 y 2007, donde el 69\% tenían la condición de empleado, el 8\% la de trabajador por cuenta propia y el $10 \%$ la de empresario.

\subsection{Formación y nivel de satisfacción}

El grado de satisfacción de los encuestados con la formación ofrecida por parte de la UMNG alcanzó el nivel de "satisfecho" para el 70,91\% y el de "totalmente satisfecho" para un 12,73\% (ver Ilustración 2). De donde se desprende que el egresado economista neogranadino en su gran mayoría encontró positiva la formación recibida a lo largo de su carrera, lo que confirman Silva \& Sarmiento (2006) para el $68,1 \%$ de los estudiantes, que manifestaron que la carrera cumplía con sus expectativas.

Ilustración 2. Grado de satisfacción con la formación ofrecida por la $\mathrm{UMNG}^{4}$

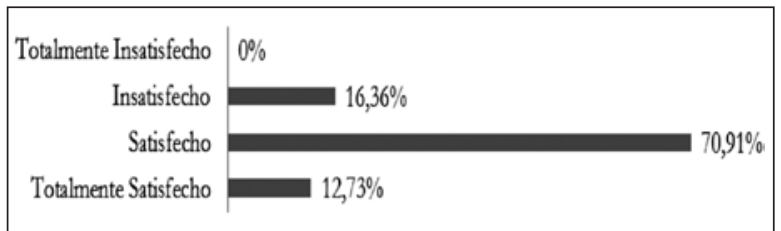

En la misma línea, para el 46\% fue "lo suficiente" el aporte de los conocimientos obtenidos y las asignaturas que constituían el plan de estudios para la proyección laboral y los proyectos de vida. En tanto que para el $10 \%$ el aporte fue "por completo". No obstante, aproximadamente un $40 \%$ de la muestra consideró "insuficiente" o "muy poco" el aporte, cifra que para Silva \& Sarmiento (2006) llegaba en los estudiantes al $31,9 \%$ por enfoque profesional y profesores.

En la tabla 1, en una escala de 1 a 5 , siendo 5 la máxima puntuación y 1 la más baja, se visualiza la calificación de las asignaturas del plan de estudios y el aporte que han tenido para los encuestados en su formación profesional.

3 Fuente: Elaboración propia.

4 Fuente: Elaboración propia. 
Tabla 1. Aporte profesional por asignatura ${ }^{5}$

\begin{tabular}{lc}
\hline \multicolumn{1}{c}{ Área } & Calificación \\
\hline Teoría microeconómica & 3,51 \\
\hline Teoría macroeconómica & 3,89 \\
\hline Estadística y econometría & 3,94 \\
\hline Análisis económico & 4,04 \\
\hline Políticas públicas & 3,42 \\
\hline Finanzas y mercado de activos & 3,69 \\
\hline Evaluación económica y social de proyectos & 3,79 \\
\hline Manejo de software estadístico y econométrico & 3,1 \\
\hline
\end{tabular}

Para la mayoría de asignaturas, el resultado de la encuesta mostró una calificación promedio de 3,5, resaltando el "análisis económico" con una media de 4,04. Por su parte, aquellas asignaturas que no fueron incluidas en el plan de estudios pero que para los egresados eran necesarias, en orden de importancia son: (i) áreas afines con las finanzas; (ii) manejo de inglés aplicado a los negocios; (iii) comercio internacional; (iv) mercado de valores; (v) manejo de software y otros. Lo que también habla del interés proyectado por los estudiantes hacia el área financiera $(44,12 \%)$, el comercio $(15,69 \%)$ y el gobierno (13,73\%) (Silva \& Sarmiento, 2006).

En prospectiva el interés de los egresados para su desarrollo profesional de mediano plazo se ubicó en áreas concernientes a ser asesor de proyectos (54,55\%), finanzas y tesorería $(36,36 \%)$, consultorías $(32,73 \%)$, investigación económica del sector público $(21,82 \%)$, investigación económica del sector privado (20\%), marketing (12,73\%), enseñanza y formación (12,73\%), y ventas $(5,45 \%)$.

Por otro lado, el instrumento de recolección de información formuló una pregunta abierta a algún comentario o sugerencia relativa a la formación recibida dentro de la UMNG, encontrando recurrentemente la necesidad de preparar economistas para el mercado de trabajo a partir del fortalecimiento del área financiera y la concepción práctica de la carrera, es decir, hacen ver la necesidad de tener una aproxima- ción mucho más constante y dirigida con la realidad económica y laboral. De igual forma manifestaron que se debía respaldar por parte de la UMNG la iniciación laboral del estudiante a partir de buenos convenios empresariales para la realización de pasantías.

\subsection{Competencia académica}

El 37,25\% consideró que la formación académica recibida en la UMNG es mejor frente a la que otros profesionales de la misma rama recibieron de otras instituciones. Es más, el 4\% piensa que es muchísimo mejor. El 31,37\% cree que es igual, en tanto que el $17,65 \%$ y el $9,8 \%$ consideran que la formación es peor o bastante inferior, respectivamente.

Ilustración 3. Formación recibida en la UMNG frente a profesionales de la misma rama graduados de distintas instituciones educativas ${ }^{6}$

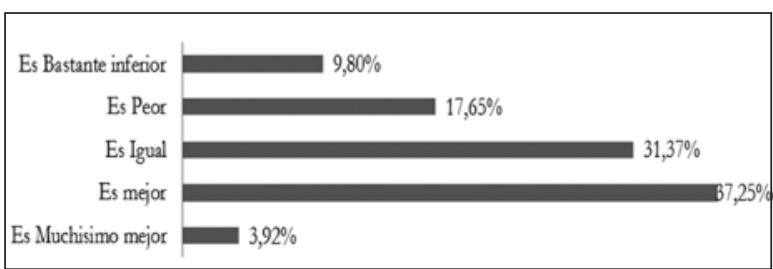

En la tabla 2, en una escala de 1 a 10 , siendo 10 la máxima puntuación y 1 la más baja, se visualiza la calificación de asignaturas que hacen parte de la formación recibida en la UMNG respecto a la que recibieron profesionales de la misma rama en otras instituciones.

Tabla 2. Contraste de asignaturas entre universidades ${ }^{7}$

\begin{tabular}{ll}
\hline \multicolumn{1}{c}{ Área } & Calificación \\
\hline Teoría microeconómica & 6,53 \\
\hline Teoría macroeconómica & 6,98 \\
\hline Estadística y econometría & 6,24 \\
\hline Análisis económico & 6,57 \\
\hline Políticas públicas & 6,16 \\
\hline Finanzas y mercado de activos & 5,86 \\
\hline Evaluación económica y social de proyectos & 6,71 \\
\hline Manejo de software estadístico y econométrico & 5,02 \\
\hline
\end{tabular}

Fuente: Elaboración propia.

Fuente: Elaboración propia.

Fuente: Elaboración propia. 
Se encontró que comparativamente existe una aceptación media de las asignaturas del plan de estudios del programa de economía de la UMNG respecto a lo que se ofrecen en otras instituciones, resaltando que "finanzas y mercado de activos" y "manejo de software estadístico y econométrico" están por debajo de la media.

\subsection{Desarrollo profesional}

De los egresados encuestados, el 55\% ya se encontraba ubicado laboralmente antes de haber recibido su título de economista, lo cual podría explicarse por los datos suministrados por Silva \& Sarmiento (2006), que indican que el 75,86\% de los estudiantes de economía de la UMNG trabajan. Por su parte el 17,5\% tardó entre 6 y 12 meses en conseguir empleo, el 15\% demoró menos de 6 meses, el 7,5\% entre 12 y 18 meses y el 5\% más de 2 años (ver Ilustración 4).

Ilustración 4. Tiempo para ubicarse laboralmente después de la obtención del título profesional ${ }^{8}$

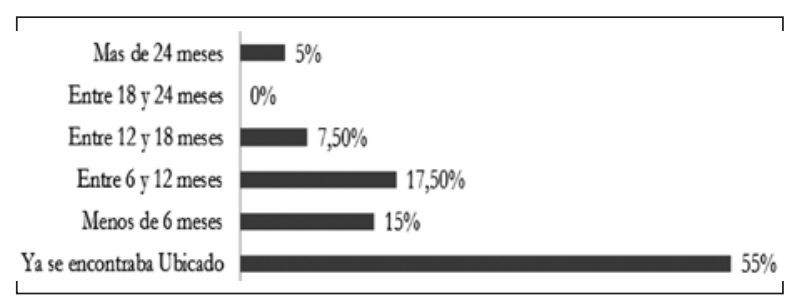

Del 80,39\% de los encuestados que manifestaron estar trabajando, el 67,5\% estaba vinculado laboralmente bajo un contrato a término indefinido. El $17,5 \%$ por contrato a término fijo y el $10 \%$ por prestación de servicios (ver Ilustración 5). En donde el 52,5\% desempeñaba un cargo como profesional, el 22,5\% como asesor, el 10\% como operativo, el $10 \%$ como directivo, y el $5 \%$ como consultor.

El 55\% se encuentra satisfecho con su desarrollo profesional dentro de su lugar de trabajo, el 15\% está totalmente satisfecho, el $27,5 \%$ se encuentra insatisfecho y el 2,5\% está totalmente insatisfecho.
Ilustración 5. Modalidad en la que se encuentran actualmente contratados ${ }^{9}$

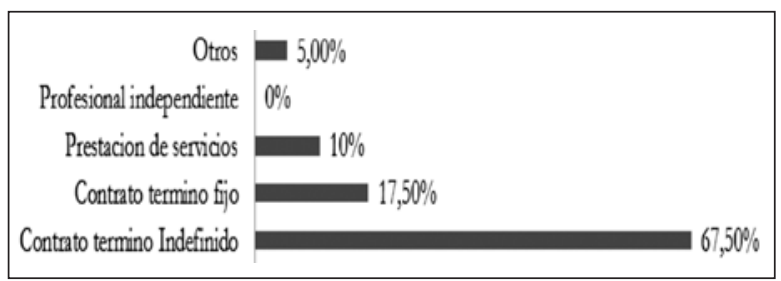

Por su parte, el rango salarial de los economistas neogranadinos encuestados estuvo en un $45 \%$ entre 1 y 2,5 millones por mes (ver tabla 3 ).

Tabla 3. Rango salarial de economistas neogranadinos ${ }^{10}$

\begin{tabular}{lc}
\hline \multicolumn{1}{c}{ Rango } & \% de personas \\
\hline Menos de \$1.000.000 & $2.5 \%$ \\
\hline Entre \$1.000.001 y \$2.500.000 & $45 \%$ \\
\hline Entre \$2.500.001 y \$ 4.000 .000 & $25 \%$ \\
\hline Entre \$ 4.000.001 y \$6.000.000 & $22,5 \%$ \\
\hline Más de \$6.000.001 & $5 \%$ \\
\hline
\end{tabular}

\subsection{Grupo Social y Empresarial de la Defensa (GSED)}

De la encuesta realizada al GSED, se presentan a continuación los resultados obtenidos en respuesta a las 4 preguntas formuladas:

- Actualmente, cuántos y en qué áreas funcionales, se encuentra laborando economistas en su empresa y de qué universidad son egresados:

$\mathrm{Al}$ interior del GSED, actualmente se encuentran laborando 46 economistas distribuidos de la siguiente manera de acuerdo al clúster al cual pertenece la entidad: 14 en Apoyo Logístico (30,46\%), 7 en Apoyo a la Seguridad (15,21\%) y 25 en Bienestar (54,34\%). De estos profesionales 6 están ubicados en áreas directivas (13,05\%), 5 en áreas administrativas (10,86\%), 10 en áreas de planeación

\footnotetext{
8 Fuente: Elaboración propia.

9 Fuente: Elaboración propia.

10 Fuente: Elaboración propia.
} 
(21,73\%), 25 en cargos operativos y/o financieros (54,34\%) -para analizar ubicación de economistas por áreas ver Ilustración 6-. Finalmente, tan solo 3 de los 46 profesionales son egresados de la UMNG, es decir, hay una participación laboral de economistas neogranadinos equivalente al $6,52 \%$ al interior del GSED.

Ilustración 6. Ocupación de profesionales economistas en el GSED ${ }^{11}$

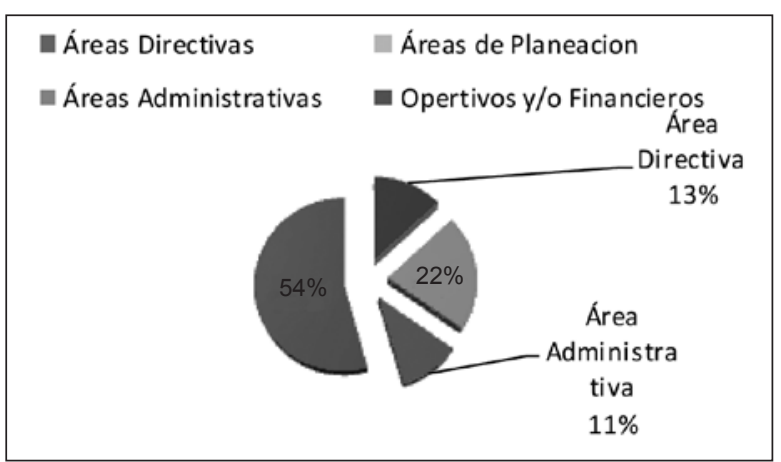

- Para qué cargos requiere específicamente la labor de un economista en su empresa y qué funciones cumple de acuerdo al manual de funciones:

En los cargos a desempeñar por un economista de acuerdo a los requerimientos del sector, sobresalen aquellos concernientes al área financiera, como jefe de grupo financiero cuyas funciones se fundamentan en el seguimiento, gestión, proyección y disponibilidad de recursos financieros. Entendiendo que el ámbito de planeación representa un área estratégica de la entidad.

Por otro lado está el jefe de la Oficina de Planeación cuyas funciones son consolidar y verificar el presupuesto de inversión de cada vigencia, elaborar los planes de acción anual y cuatrienal, proponer y formular el direccionamiento estratégico de corto y mediano plazo y la visión prospectiva de largo plazo, proponer las metas MEGA de la entidad, dirigir, liderar, coordinar y asesorar en la elaboración del proyecto de presupuesto de funcionamiento e inversión, distribuyendo excedentes financieros y elaborando el programa anual de caja. Algunos otros cargos están en representación del área administrativa, en control interno, operaciones, riesgos y análisis estadísticos.

Además los economistas pueden hacer parte del comité económico, dentro del cual deben efectuar evaluación económica, gestión de respuestas rápidas, participación activa de audiencias, verificación y control de la información, análisis y elaboración del anteproyecto de presupuesto para cada vigencia de funcionamiento e inversión, gestión de la ejecución del presupuesto aprobado para cada vigencia, desarrollo de estrategias que garanticen la administración eficiente de los recursos económicos y financieros de la entidad, y verificación periódica de los procesos, elaboración de información financiera y asesoramiento a la alta dirección sobre la toma de decisiones en materia financiera.

- En su concepto, cuáles son las competencias que debe tener un economista de acuerdo con la misión y el objeto social que maneja su empresa:

Aquellas competencias que reflejan las necesidades de demanda laboral por parte del GSED fueron planteadas a partir de aptitudes profesionales, que en su mayoría están ligadas al área de finanzas, y competencias genéricas que aluden a la capacidad integral del profesional en su análisis y pensamiento, de tal forma que se plantea el perfil del economista a partir del control, planeación, rendimiento de informes, gestión y administración financiera, planeación y formulación de proyectos, habilidades estadísticas, proyectos y portafolios de inversión.

De igual forma se propende por la capacidad analítica e interpretativa, propositiva y argumentativa, de análisis y síntesis, pensamiento estratégico, comunicación asertiva, adaptación al cambio, resolución de problemas, fluidez verbal, toma de decisiones, visión global, trabajo bajo presión, desarrollo creativo y capacidad innovadora, prospectiva, trabajo en equipo, pensamiento sistémico y complejo.

Si usted fuera a seleccionar un economista para una posición especifica dentro de la organización, con qué otra profesión lo haría equivalente:

11 Fuente: Elaboración propia a partir de información suministrada por GSED. 
Las carreras equivalentes al programa de economía son varias, por afinidad con las ciencias económicas estarían la administración pública, la administración financiera, la administración de empresas, la ingeniería financiera, la ingeniería industrial, las especializaciones en finanzas, las matemáticas y la contaduría pública. En cuyo caso, los profesionales de tales carreas son funcionalmente equivalentes.

\subsection{Consejo de Empresas Americanas (CEA)}

De la encuesta realizada al CEA, se presentan a continuación los resultados obtenidos en respuesta a las 4 preguntas formuladas:

- Para qué cargos requiere la labor de un economista en su empresa y qué funciones cumple:

Los resultados concuerdan con los informes de los egresados y el GSED, enfocando prioritariamente el perfil de un economista en áreas asociadas al control, gestión y desarrollo financiero, al igual que aquellas fundamentadas en la planeación y gestión de proyectos. Sin embargo, existen otros ámbitos en el campo de acción del economista en el CEA evidenciados a partir de la prestación de servicios en asesorías, consultorías y en departamento de negocios y ventas. De manera general se requiere un profesional fuertemente capacitado en aptitudes analíticas, estratégicas y tácticas.

- Cuáles son las competencias que debe tener el economista en su empresa:

Las competencias expuestas por el CEA son: (i) comunicativas, dentro de las cuales se requiere el trabajo en equipo, la comunicación efectiva la flexibilidad, el manejo del estrés y el aprendizaje; (ii) propositivas, tales como la gestión de resultados, versatilidad y mejora de procesos; y (iii) analíticas, condensadas en el análisis y estrategia, visión de negocio y lectura del contexto económico global.
- Cuáles son las funciones del economista:

Dentro del manual de funciones de cada entidad perteneciente al CEA no existen funciones definidas y específicas para un economista, éstas se establecen de acuerdo al cargo desempeñado, que como se mencionaba con anterioridad, dependen en su gran mayoría del área financiera, de planeación y de proyectos, dentro de las cual se especifican funciones referentes al análisis de cifras, manejo de información, proyección de crecimiento y manejo de presupuestos.

- Si fuera a seleccionar un economista para una posición especifica dentro de la organización con qué otra profesión lo haría equivalente y de qué universidad lo contrataría:

Con referencia a los profesionales que de forma equivalente podrían sustituir a un economista, el CEA coincide con la respuesta del GSED. Además anota que la demanda de egresados se hace regularmente de instituciones con reconocimiento social como la Universidad de los Andes, la Javeriana, la Sabana, el Rosario, la Nacional y el CESA.

\section{Discusión de resultados}

La información obtenida de las encuestas efectuadas a los egresados economistas de la UMNG, al GSED y el CEA, arrojó coincidencias significativas en torno a las necesidades y requerimientos que el mercado laboral hace teniendo en cuenta aspectos fundamentales como la inserción de asignaturas relevantes, la inclusión laboral, el manejo de conocimientos específicos, y en términos generales, la consolidación de economistas fuertemente instruidos en competencias argumentativas, propositivas, interpretativa y analíticas.

Intentando establecer paralelos entre la información suministrada por el GSED y el CEA, se hace el comparativo de la Tabla 4. 
Tabla 4. Comparativo GSED - CEA ${ }^{12}$

\begin{tabular}{|c|c|c|}
\hline & GSED & CEA \\
\hline Cargos del Economista & $\begin{array}{l}\text { Cargos directivos (13,05\%) } \\
\text { Áreas administrativas }(10,86 \%) \\
\text { Áreas de planeación }(21,73 \%) \\
\text { Cargos financieros }(43,47 \%)\end{array}$ & $\begin{array}{l}\text { Áreas financieras } \\
\text { Áreas de planeación } \\
\text { Gestión de proyectos }\end{array}$ \\
\hline Funciones del economista & $\begin{array}{l}\text { Seguimiento financiero } \\
\text { Gestión financiera } \\
\text { Proyección financiera } \\
\text { Disponibilidad financiera } \\
\text { Consolidación de presupuestos } \\
\text { Direccionamiento estratégico } \\
\text { Elaboración de proyectos } \\
\text { Control interno } \\
\text { Análisis de riesgos } \\
\text { Análisis estadísticos } \\
\text { Evaluación Económica } \\
\text { Consultorías/asesorías }\end{array}$ & $\begin{array}{l}\text { Análisis de cifras } \\
\text { Gestión de proyectos } \\
\text { Manejo de información } \\
\text { Proyección de crecimiento } \\
\text { Manejo de presupuestos } \\
\text { Control financiero }\end{array}$ \\
\hline Competencias & $\begin{array}{l}\text { Capacidad analítica: } \\
\text { Análisis estadístico } \\
\text { Análisis estratégico } \\
\text { Capacidad interpretativa: } \\
\text { Manejo de información } \\
\text { Administración de recursos } \\
\text { Capacidad propositiva: } \\
\text { Gestión de Proyectos - Planeación } \\
\text { financiera - Gestión de Resultados } \\
\text { Capacidad comunicativa: } \\
\text { Excelente expresión oral } \\
\text { Versatilidad }\end{array}$ & $\begin{array}{l}\text { Capacidad analítica: } \\
\text { Análisis estrategia } \\
\text { Visión de negocios } \\
\text { Análisis económico. } \\
\text { Capacidad interpretativa: } \\
\text { Aprendizaje } \\
\text { Manejo de información } \\
\text { Capacidad propositiva: } \\
\text { Gestión de resultados } \\
\text { Mejoramiento de procesos } \\
\text { Capacidad comunicativa: } \\
\text { Trabajo en equipo - Comunicación efectiva } \\
\text { Flexibilidad - Manejo de estrés }\end{array}$ \\
\hline Competencia equivalente & $\begin{array}{l}\text { Profesiones: } \\
\text { Administradores Públicos } \\
\text { Administradores de Empresas } \\
\text { Ingenieros Industriales } \\
\text { Ingenieros Financieros } \\
\text { Matemáticos } \\
\text { Contadores públicos } \\
\text { Especialistas en Finanzas } \\
\text { Universidades: } \\
\text { Andes } \\
\text { Javeriana } \\
\text { Rosario } \\
\text { Nacional }\end{array}$ & $\begin{array}{l}\text { Profesiones: } \\
\text { Administradores Públicos } \\
\text { Administradores de Empresas } \\
\text { Ingenieros Industriales } \\
\text { Ingenieros Financieros } \\
\text { Contadores públicos } \\
\text { Especialistas en Finanzas } \\
\text { Universidades: } \\
\text { CESA } \\
\text { Andes } \\
\text { Javeriana } \\
\text { Rosario } \\
\text { Nacional } \\
\text { Sabana }\end{array}$ \\
\hline
\end{tabular}

12 Fuente: elaboración propia a partir de información suministrada por GSED y CEA. 


\section{1. Áreas de conocimiento: finanzas, inglés y planeación}

Existe un área de conocimiento propia de la economía que el mercado laboral requiere constantemente en el perfil del profesional. Según el GSED, el CEA y los egresados, las finanzas se muestran como un rubro fundamental en la formación académica del estudiante, pues constantemente en los procesos directivos, administrativos u operacionales de las entidades se considera preponderante la presencia de un profesional idóneo con relación al manejo, gestión, dirección, proyección, seguimiento y disponibilidad de recursos financieros.

Se pensaría entonces, en la inclusión de finanzas dentro del pensum académico a partir de distintas perspectivas como análisis de riesgos, finanzas corporativas, mercado de valores, gestión y proyección. Aunque si bien en este momento el programa de economía de la UMNG ofrece asignaturas electivas y del plan de estudios que guardan alguna afinidad con las finanzas, se ve la necesidad de fortalecer tal área de formación, lejos de la forma en que lo perciben actualmente los egresados, es decir, como una parte de conocimiento aislado que debe equipararse en importancia a la macroeconomía, microeconomía y estadística.

De igual forma, el segundo idioma (inglés) es considerado por todas las partes (egresados, GSED y CEA) como un innegable diferenciador del profesional en economía. En efecto, es una falencia frecuente que se identifica en los egresados, que puede ser resuelta mediante lecturas y materiales en inglés que sean parte de todas y cada una de las asignaturas, de tal forma que la frecuencia y repetición del inglés se convierta en un hábito estudiantil, lo cual se puede ser reforzado con cursos que estén dentro el plan de estudios, cuya orientación sea hacia el inglés corporativo o de negocios.

Igualmente, la evidencia mostró que existen unas demandas particulares por conocimientos específicos de la estadística. El manejo de software especializado es un valor agregado totalmente necesario y apreciado por el mercado laboral. La inconformidad del egresado al respecto es frecuente a la luz de una competencia fuerte. Por ello, el perfil neogranadino debe incluir la operación especializada de paquetes estadísticos y econométricos (Eviews, Rats, Spss, Stata, entre otros) y de proyectos (Project Management).

Por último, la demanda del GSED y el CEA por economistas que trabajen en el área de planeación está latente, para la ejecución de funciones específicas que han venido estando en manos de profesionales de administración e ingenierías. En tal área, las funciones se orientan a la consolidación y verificación de presupuestos de inversión, la elaboración de planes de acción, la proposición del direccionamiento estratégico, la formulación de metas de la entidad, la dirección, liderazgo, coordinación y asesoramiento en la elaboración del proyecto de presupuesto de funcionamiento e inversión de una empresa, además de la distribución de excedentes financieros y programa anual de caja.

\section{Consideraciones finales}

En consideración a la posición del egresado neogranadino, hubo algunas solicitudes respecto a la metodológica empleada por la UMNG, teniendo en cuenta que si bien la fundamentación teórica debe seguir un lineamiento riguroso, la contextualización práctica del conocimiento se hace necesaria para la comprensión global y efectiva de las asignaturas en su conjunto, de tal manera que el horario cotidiano de cada clase brinde un espacio esencial e inmovible para el manejo práctico a partir de las facilidades de contenido de cada asignatura.

Este requerimiento se hace teniendo en cuenta la brecha existente entre lo enseñado en el aula de clase y lo requerido por el mercado laboral, en otras palabras, la conciliación entre la teoría y la práctica. Por ello, es imperioso el empalme del acervo teórico con la realidad económica nacional y mundial.

En virtud de esta solicitud, se espera la formación de economistas neogranadinos cada vez más competentes en el análisis económico, aspecto igualmente valorado por el mercado laboral. Se entiende que este desarrollo de habilidades viene precedido por el incentivo a la formación en investigación. 
Otra de las grandes solicitudes que aqueja el egresado y que consideran debe tener un trato prioritario, es la inclusión laboral del estudiante a través de convenios institucionales importantes que le permitan iniciar su vida laboral por medio de las prácticas empresariales, lo que le brinda oportunidades y lo prepara para su desarrollo profesional.

En la actualidad, la mayoría de los estudiantes buscan aquellos convenios por su cuenta, encontrándose por ejemplo, que no existen convenios interinstitucionales con entidades del mercado bursátil (área importante del economista), Proexport, el Ministerio de Hacienda y otras entidades públicas y privadas. Es común que los requerimientos administrativos para realizar las prácticas con otras entidades sea desmotivante. Encontrando en muchos casos que existe demérito de la formación profesional y laboral del egresado economista de la UMNG.

Por otra parte, uno de los aspectos a tener en cuenta respecto de las falencias y necesidades del egresado neogranadino, es el resultado obtenido del promedio salarial de la muestra, el cual evidencia que éste se encuentra en el segundo quintil más bajo. La situación demanda atención a sabiendo de que más del 60\% de la muestra se ubica en edades superiores a los 31 años de edad.

En lo que tiene que ver con el GSED y el CEA, es conveniente resaltar que tan solo 3 de los 46 profesionales economistas que trabajan en el GSED son egresados de la UMNG, es decir, hay una leve participación laboral de economista neogranadinos equivalente al 6,52\%, dato no menos importante, considerando el vínculo de la UMNG con el sector defensa. De modo que existe en el GSED un campo laboral inexplorado, entendiendo que allí se demandan egresados aptos para el área financiera y de planeación, además de profesionales en el comité económico.

En lo que respecta al CEA, se evidencia también la necesidad de formar y preparar economistas fuertemente instruidos en el área de finanzas, planeación y gestión de proyectos. El perfil profesional aboga por aquellas actitudes y aptitudes que demuestren eficiencia, trabajo en equipo, gestión de resultados, versatilidad, proyección de crecimiento, análisis, táctica, visión de negocios y mejoramiento de procesos. Más aun, profesionales idóneos que aporten y brinden su conocimiento y formación en función del crecimiento estratégico de las entidades.

Por su parte, las competencias como elemento que ha transformado la educación, específicamente en la formación de profesionales, son pues, una medida del éxito laboral de los egresados en la economía de mercado. Son las competencias específicas y genéricas la llave de acceso al mundo laboral. Entendiendo las primeras como las habilidades propias de la carrera que se convierten en factor diferenciador, y que en el caso de los egresados de economía de la UMNG deben afinarse para aprovechar la cercanía con el sector defensa.

En tanto que las competencias genéricas sirviendo de manera más general para enfrentar el mundo laboral, deben propender por la capacidad analítica, interpretativa, propositiva y argumentativa, de análisis y síntesis, pensamiento estratégico, comunicación asertiva, adaptación al cambio, resolución de problemas, fluidez verbal, toma de decisiones, visión global, trabajo bajo presión, desarrollo creativo y capacidad innovadora, prospectiva, trabajo en equipo, pensamiento sistémico y complejo.

Ahora bien, los comentarios y sugerencias que los egresados hicieron con el fin de fortalecer la estructura del programa de Economía, se pueden resumir en cuatro: (i) la inserción laboral del estudiante desde la universidad, (ii) la profundización constante del segundo idioma -inglés-, (iii) el acercamiento práctico de cada área a la realidad económica y laboral, y (iv) la inclusión de asignaturas referentes a las finanzas, comercio internacional y manejo de software especializados.

Para finalizar, dentro de una futura agenda de investigación sobre el tema, valdría la pena profundizar sobre los vínculos UMNG y GSED, de cara a la inserción laboral de graduados neogranadinos. 


\section{Referencias}

Aguila, V. (2005). El concepto de calidad en la educación universitaria: Clave para el logro de la competitividad institucional. En: Revista Iberoamericana de educación, OIE, No. 35/5.

ANECA (2009) Los procesos de inserción laboral de los titulados universitarios en Espeña. Factores de facilitación y obstaculización. Agencia nacional de evaluación de la calidad y acreditación -ANECA-, Madrid. Disponible en: www.aneca.es/ content/download/10357/115911/.../publi_procesosil.pdf

Beneitone, P. et al. (2007) Reflexiones y perspectivas de la educación superior en América Latina: informe final - proyecto Tuning - América Latina 2004-2007. Bilbao: Universidad de Deusto.

Calderón, M.; Ríos, M. \& Ceccarini, M. (2008) Economía de la educación. Universidad Nacional de Cuyo, Mendoza.

Cardoso, C. \& Giraldo, C. (2003) Mercado laboral y formación por competencias. Conceptos para una mirada analítica de las competencias laborales. Monografía para proyecto de grado. Carrera de Economía, Universidad Nacional de Colombia. Disponible en: http://www.bdigital.unal.edu.co/2148/1/ MERCADO_LABORAL_Y_FORMACION_POR_COMPETENCIAS.pdf

Carlson, B. (2000) ¿Cómo lograr una educación de calidad: Qué nos enseñan las escuelas? ¿Qué podemos aprender de las escuelas básicas del programa P900 de Chile? Red de Reestructuración y competitividad de la Cepal, Naciones Unidas. Eclac. Santiago de Chile.

Duque, Y. \& Ortíz, C. (2011). La actividad empresarial del graduado neogranadino. En: Revista Facultad de Ciencias Económicas de la Universidad Militar Nueva Granada, rev. fac.cienc.econ, XIX (1).

Flores, H. (2007). Proyecto Tuning América Latina. Reflexiones y Perspectivas de la Educación superior en América Latina. Informe Final 2004-2007, Universidad Deusto-Universidad de Groningen, Bilbao.

Gallard, M. (1995) El desafío de la formación para el trabajo de jóvenes en situación de pobreza: El caso argentino. Centro de estudios de población CENEP. Buenos Aires.
García-Montalvo, J. (2005) Los graduados universitarios y el mercado laboral: el ajuste temporal y competencial entre egresados y puestos de trabajo. Mimeo.

Lora, E. \& Ñopo, H. (2009). La formación de los economistas en América Latina. En: Revista de Análisis Económico, 24 (2): 65-93.

Márquez, P. (2005). 5 claves para una buena integración de las TIC en los centros docentes. Departamento de pedagogía aplicada. Universidad Autónoma de Barcelona.

Periáñez, et al. (2010) Competencias demandadas en los nuevos economistas. En: Revista de Educación en Contabilidad, Finanzas y Administración de Empresas, 1(1): 59-77.

Pérez, J. (2010) Competencias laborales de los egresados de la licenciatura en economía de la UADY en ejercicio profesional. Tesis de grado. Maestría en Investigación Educativa -Universidad Autónoma de Yucatán. Disponible en: http:// posgradofeuady.org.mx/wp-content/uploads/2011/01/ Perez-Jenifer-MIE-2010.pdf

SENA-Servicio Nacional de Aprendizaje (2003) Sistema nacional de formación para el trabajo, enfoque colombiano. SENA, Bogotá - Colombia.

Schomburg, H. (2004) Manual para estudios de seguimiento de graduados universitarios. Centro para la investigación sobre la educación superior y el trabajo. Universidad de Kassel, Alemania.

Silva, A. \& Sarmiento, J. (2006) Estudiantes de Economía de la UMNG, ¿Cómo son? En: Revista Facultad de Ciencias Económicas de la Universidad Militar Nueva Granada, rev. fac.cienc.econ, XIV (1): 238-257.

Tedesco, J. et al. (2008). Las TIC: del aula a la agenda política. Ponencias del seminario internacional "Cómo las TIC transforman las escuelas”. IIPE-UNESCO/UNICEF. Sede regional Buenos Aires.

Vasconez, A. \& Trujillo, A. (2004). Incorporación de los jóvenes al mercado laboral. FLACSO sede Ecuador. Disponible en: http:// www.eclac.cl/de/noticias/paginas/2/14692/FLACSO.pdf

Von Mises, L. (2009) La acción humana: tratado de economía. Unión Editorial.

Zúñiga, G. (2003) La oferta laboral en Venezuela. Una perspectiva regional de los últimos veinte años. En: Revista Temas de Coyuntura, No. 47. 